\title{
STRONGLY ANNULAR TSUJI FUNCTIONS
}

\author{
C. L. BELNA, D. D. BONAR, F. W. CARROLL, \\ P. COLWELL AND G. PIRANIAN
}

\section{Annular functions}

A holomorphic function $f$ in the unit disk $D$ is annular if there exists a nested sequence $\left\{J_{n}\right\}$ of Jordan curves surrounding the origin such that each annulus $\{z: 1-\varepsilon<|z|<1\}$ contains infinitely many of the curves $J_{n}$ and such that the minimum of $|f(z)|$ on $J_{n}$ tends to $\infty$ as $n \rightarrow \infty$. The function $f$ is strongly annular if for $\left\{J_{n}\right\}$ we can choose a sequence of concentric circles.

\section{Tsuji functions and Julia points}

A holomorphic or meromorphic function $f$ in $D$ is a Tsuji function if in the interval $0<r<1$ the spherical length $L(r)=L(r, f)$ of the image of the circle $\gamma_{r}=\{z:|z|=r\}$ is a bounded function of $r$.

If $f$ is meromorphic in $D$, if $S$ is a line segment lying in $D$ except for one endpoint, and if in each Stolz angle bisected by $S$ the function $f$ assumes every value in the extended plane, with at most two exceptions, then $S$ is a segment of Julia for $f$. If each segment in $D$ terminating at the point $e^{i \theta}$ is a segment of Julia for $f$, then $e^{i \theta}$ is a Julia point for $f$.

In [4], E. F. Collingwood and Piranian showed that the set of Julia points of a meromorphic Tsuji function may consist of the entire unit circle. They also showed that the holomorphic function

$$
f(z)=\exp \left(\frac{1+z}{1-z}\right)^{2}
$$

is a Tsuji function with two segments of Julia, and they conjectured that a holomorphic Tsuji function can have at most finitely many segments of Julia. In [6], W. K. Hayman disproved this conjecture by constructing a holomorphic Tsuji function with a countably infinite set of Julia points. We shall extend Hayman's result by showing that the set of Julia points of a holomorphic Tsuji function may consist of the entire unit circle.

\section{Strongly annular functions}

The question whether a Tsuji function can be annular has circulated for several years (its first recorded discussion occurs in [1; Section 4]). We shall consider the problem in the context of strong annularity.

A natural way of constructing a strongly annular function is to choose a rapidly increasing sequence $\left\{k_{n}\right\}$ of positive integers and to define

$$
f(z)=\sum k_{n} z^{k_{n}}
$$

Received 28 May, 1977; revised 24 April, 1978.

F. W. Carroll acknowledges support from the National Science Foundation.

[J. LoNDON MATH. Soc. (2), 19 (1979), 79-92] 
Because on the circle $|z|=1-1 / k_{n}$ the $n$th term dominates and its modulus is approximately $k_{n} / e$, the function $f$ is strongly annular. Because $f$ maps the circle $|z|=1-1 / k_{n}$ onto a curve that encircles the origin $k_{n}$ times and at a distance approximately $k_{n} / e$, the spherical length $L\left(1-1 / k_{n}, f\right)$ is approximately $k_{n}\left(2 \pi e / k_{n}\right)=2 \pi e$. Nevertheless, $f$ is not a Tsuji function; for on a certain circle of radius slightly greater than $1-1 / k_{n}$, the $n$th and $(n+1)$ st term in the series for $f$ have equal modulus. The image on the Riemann sphere of that circle consists of many loops of sufficiently large diameter so that the length of the curve is large.

These considerations suggest that strong annularity and bounded spherical length might be incompatible. However, it has been known for some time that neither lacunarity nor unboundedness of the Taylor coefficients is essential for strong annularity. R. W. Howell [8] has shown that under the topology of locally uniform convergence, a residual subset of the space of functions $\Sigma \varepsilon_{n} z^{n}\left(\varepsilon_{n}= \pm 1\right)$ consists of strongly annular functions. Also, Bonar, Carroll, and Piranian [2] have proved that if the sequence $\left\{k_{n}\right\}$ increases rapidly enough, then the function

$$
f(z)=\sum_{n=1}^{\infty} \frac{k_{n}^{1 / 3}}{n^{1 / 2}}\left(\frac{z-1 / 2}{1-z / 2}\right)^{k_{n}}
$$

is strongly annular, although the sequence of its Taylor coefficients at the origin tends to 0 .

Aware that strongly annular functions are more abundant than we had originally believed, we undertook an intensive search for a strongly annular Tsuji function. The example developed in Sections 4 to 13 resembles the function $f$ given in the preceding paragraph; but there are two noticeable differences: In the new example, the ratio between the coefficient and the exponent in the $n$th term is much larger, and the sequence $\left\{k_{n}\right\}$ of exponents is subject to careful control.

In Section 14, we use a recent result of S. Dragosh [5] to obtain a theorem on the union of the sets of Julia points and points of spherical continuity of functions in Hayman's class $T_{2}$ (this class is substantially larger than the class of Tsuji functions).

\section{The main result}

THEOREM 1. There exists a strongly annular Tsuji function whose set of Julia points is the unit circle.

To prove the theorem, we shall construct a function

$$
f(z)=\sum a_{n} z^{k_{n}}
$$

such that, for each Möbius transformation

$$
g_{b}(z)=\frac{z-b}{1-b z} \quad(0<|b|<1),
$$

the composite function $f_{b}=f \circ g_{b}$ is a strongly annular Tsuji function. That each point $e^{t \theta}$ is a Julia point for $f$ and for each $f_{b}$ will be a trivial consequence of the construction. 


\section{The coefficients and exponents}

The motivation for our choice of the coefficients $a_{n}$ and the exponents $k_{n}$ in (1) will become apparent in our later computations. At this stage, we merely plead the need for rapid growth of the sequence $\left\{k_{n}\right\}$ and for a carefully adjusted relation between $\left\{a_{n}\right\}$ and $\left\{k_{n}\right\}$. If the sequence $\left\{k_{n}\right\}$ grows too slowly, the annuli in which two consecutive terms of the series (1) have approximately equal modulus are not sufficiently separated, and unless we choose the coefficients $a_{n}$ with proper care, the troublesome annuli are too broad for our purposes.

We set $k_{1}=h_{1}=1$, and we defer until later the choice of the coefficient $a_{1}$. For $n=2,3, \ldots$, we define

$$
\begin{aligned}
& h_{n}=a_{n-1} / h_{n-1}, \\
& a_{n}=\exp h_{n} / h_{n-1} ;
\end{aligned}
$$

for each index $n$, we set $k_{n}=\left[h_{n}\right]$ (greatest-integer notation). A cursory estimate shows that if $a_{1} \geqslant 7$, the sequences $\left\{a_{n}\right\}$ and $\left\{k_{n}\right\}$ grow rapidly; but we need a quantitative formulation of this.

Lemma 1. If $p=2,3, \ldots$ and $a_{1} \geqslant(p+6)$ !, then $h_{n}>h_{n-1}^{p}$ for $n=2,3, \ldots$.

Proof. Since $h_{1}=1$ and $h_{2}=a_{1}$, the inequality in the lemma is obviously satisfied for $n=2$. Suppose it holds for some integer $n$. By condition (3), the inequality then implies that

$$
a_{n}>\exp h_{n}{ }^{1-1 / p} \text {, }
$$

and from the power series expansion of the exponential function we deduce that

$$
a_{n}>\frac{h_{n}^{(1-1 / p) j}}{j !}
$$

for each natural number $j$. From (2) it follows further that

$$
h_{n+1}>\frac{1}{j !} h_{n}^{(1-1 / p) j-1} .
$$

For the special choice $j=p+6$, the exponent on the right is not less than $p+1$, and if in addition $h_{n}>j$ !, it follows that $h_{n+1}>h_{n}{ }^{p}$. In particular, if we choose $a_{1}=j !=(p+6)$ !, the required inequality holds for all indices $n$.

\section{One-term dominance}

On the circle $|z|=r_{n}=1-1 / h_{n}$, the value of $|z|^{k_{n}}$ is approximately $1 / e$; therefore the modulus of the $n$th term in (1) is approximately $a_{n} / e$. We shall show that this is much larger than the sum of all earlier and all later terms.

By (2), $a_{m}=h_{m} h_{m+1}$, and by Lemma 1, we may assume that

$$
\frac{a_{m}}{a_{m-1}}>\frac{h_{m}{ }^{p+1}}{h_{m} h_{m-1}}=h_{m}{ }^{p} / h_{m-1},
$$


where $p$ is some integer greater than 1 . Therefore the sequence $\left\{a_{m}\right\}$ increases so rapidly that

$$
a_{1}+\ldots+a_{n-1}=o\left(a_{n} / e\right) .
$$

To estimate the later terms, we note that

$$
a_{m} r_{n}^{k_{m}}=\exp \left(\frac{h_{m}}{h_{m-1}}+k_{m} \log \left(1-\frac{1}{h_{n}}\right)\right)<\exp \left(\frac{h_{m}}{h_{m-1}}-\frac{h_{m}-1}{h_{n}}\right) .
$$

If $m=n+1$, the last expression reduces to $\exp \left(1 / h_{n}\right)$, and this is less than 3. If $m>n+1$, we use the inequality $h_{m} / h_{m-1}<h_{m} / 3 h_{n}$ and deduce that

$$
a_{m} r_{n}^{k_{m}}<\exp \left(1-h_{m} / 2 h_{n}\right)
$$

Invoking Lemma 1 again, we see that

$$
f\left(r_{n} e^{i \theta}\right) \sim a_{n} e^{i k_{n} \theta-1} .
$$

\section{The dirty annuli}

In Section 6, we saw that on the circle $|z|=r_{n}=1-1 / h_{n}$, the $(n+1)$ st term of the series in (1) has modulus less than 3 . We shall now examine the modulus of the same term on the slightly larger circle $|z|=r_{n}+1 / h_{n}{ }^{2}$. By elementary computations,

$$
\begin{aligned}
& a_{n+1}\left(1-1 / h_{n}+1 / h_{n}{ }^{2}\right)^{k_{n+1}} \geqslant a_{n+1}\left(1-\frac{1}{h_{n}}\left(1-\frac{1}{h_{n}}\right)\right)^{h_{n+1}} \\
= & \exp \left\{\frac{h_{n+1}}{h_{n}}+h_{n+1} \log \left(1-\frac{1}{h_{n}}\left(1-\frac{1}{h_{n}}\right)\right)\right\} \\
= & \exp \left\{h_{n+1}\left[\frac{1}{h_{n}}-\frac{1}{h_{n}}\left(1-\frac{1}{h_{n}}\right)-\frac{1}{2 h_{n}{ }^{2}}\left(1-\frac{1}{h_{n}}\right)^{2}-\ldots\right]\right\} \\
\sim & \exp h_{n+1} / 2 h_{n}{ }^{2} .
\end{aligned}
$$

By Lemma 1, we may assume that $h_{n+1}>3 h_{n}{ }^{3}$, so that on the circle $|z|=r_{n}+1 / h_{n}{ }^{2}$ the $(n+1)$ st term in (1) has modulus greater than $\exp h_{n}$. By (3), this is much greater than $a_{n}$, and therefore the $(n+1)$ st term dominates the series, on the circle

We call the annulus

$$
|z|=r_{n}+1 / h_{n}{ }^{2} .
$$

$$
r_{n}<|z|<r_{n}+1 / h_{n}{ }^{2}
$$

the $n$th dirty annulus, and we observe that each dirty annulus is narrow, compared with its distance from the unit circle. Because the $(n+1)$ st term of $(1)$ dominates the sum of all other terms, both on the outer boundary component of the $n$th dirty annulus and on the inner boundary component of the $(n+1)$ st dirty annulus, and because the $(n+1)$ st term has no zeros except at the origin, we can assert that

$$
f(z) \sim a_{n+1} z^{k_{n+1}}
$$

in the domain between the dirty annuli. Consequently, $f$ is strongly annular. 


\section{Strong annularity of the function $f_{b}$}

If $b=\beta e^{i \phi}$, then the function $g_{b}(z)=(z-b) /(1-\bar{b} z)$ attains its maximum and minimum moduli on the circle $|z|=r$ at the points $z=-r e^{i \phi}$ and $z=r e^{i \phi}$, and the two moduli are

$$
\frac{r+\beta}{1+r \beta}=1-(1-r) \frac{1-\beta}{1+r \beta}
$$

and

$$
\frac{|r-\beta|}{1-r \beta}=\left|1-(1-r) \frac{1+\beta}{1-r \beta}\right| .
$$

If $r$ is near 1 , the coefficients of $1-r$ in the two expressions are approximately

$$
(1-\beta) /(1+\beta) \quad \text { and } \quad(1+\beta) /(1-\beta),
$$

respectively. In particular, the function $g_{b}$ maps the circle $|z|=1-1 / h_{n}{ }^{2}$ onto a circle lying between the $n$th and $(n+1)$ st dirty annuli. By the argument in Section 7 , each function $f_{b}$ is strongly annular.

\section{The image of the circle $|z|=r$}

For each value $b$ in $D$, the function

$$
f_{b}(z)=\sum a_{n}\left(\frac{z-b}{1-b_{z}}\right)^{k_{n}}
$$

maps each circle $\gamma_{r}=\{z:|z|=r\}$ onto a more or less convoluted curve $\Gamma_{r}$. We shall devote several sections to the task of showing that the spherical length $L\left(r, f_{b}\right)$ of $\Gamma_{r}$ is a bounded function of $r$.

The analysis in Sections 6 and 7 shows that on the circle $|z|=r_{n}$ the $n$th term of the series in (1) is much larger than the sum of all the other terms, while on the circle $|z|=r_{n}+1 / h_{n}{ }^{2}$ the $(n+1)$ st term is much larger than the sum of all the other terms. Modifying the arguments slightly, we shall now establish similar one-term dominance for the power series of $f^{\prime}$.

On the circle $|z|=r_{n}=1-1 / h_{n}$, the $n$th term in the differentiated series has the absolute value

$$
a_{n} k_{n} r_{n}^{k_{n}-1} \sim a_{n} h_{n} / e=h_{n}{ }^{2} h_{n+1} / e
$$

(the equality is a consequence of (2)). The sum of the preceding terms has absolute value less than

$$
a_{1} k_{1}+a_{2} k_{2}+\ldots+a_{n-1} k_{n-1} \sim a_{n-1} h_{n-1}=h_{n-1}^{2} h_{n},
$$

and clearly the $n$th term dominates the sum of the earlier terms. To estimate the later terms, we use the computations in Section 6 and obtain the bounds

and (for $m>n+1$ )

$$
a_{n+1} h_{n+1} r_{n}{ }^{k_{n+1}}<3 h_{n+1}=o\left(h_{n}{ }^{2} h_{n+1}\right)
$$

$$
\begin{aligned}
h_{m} a_{m} r_{n}{ }^{k_{m}} & <\exp \left(1+\log h_{m}-h_{m} / 2 h_{n}\right) \\
& <\exp \left(-\sqrt{ } h_{m}\right) .
\end{aligned}
$$


We conclude that $f^{\prime}(z) \sim a_{n} k_{n} z^{k_{n}-1}$, on the circle $|z|=r_{n}$.

On the circle $|z|=r_{n}+1 / h_{n}{ }^{2}$, the sum of the first $n$ terms is less than

$$
a_{1} h_{1}+\ldots+a_{n} h_{n} \sim a_{n} h_{n}=h_{n}^{2} h_{n+1} .
$$

For the $(n+1)$ st term, the computations in Section 7 give

$$
a_{n+1} h_{n+1}|z|^{h_{n+1}} \sim h_{n+1} \exp \left(h_{n+1} / 2 h_{n}{ }^{2}\right)=\left(a_{n} / h_{n}\right) \exp \left(h_{n+1} / 2 h_{n}{ }^{2}\right) .
$$

Because we may assume that $h_{n+1}>h_{n}{ }^{3}$, this is much larger than the sum of the first $n$ terms. Finally, for the terms with indices $m>n+1$, we use the estimate

$$
\begin{aligned}
a_{m} h_{m}\left[1-\frac{1}{h_{n}}\left(1-\frac{1}{h_{n}}\right)\right]^{h_{m}} & =\exp \left\{\frac{h_{m}}{h_{m-1}}+\log h_{m}+h_{m} \log \left[1-\frac{1}{h_{n}}\left(1-\frac{1}{h_{n}}\right)\right]\right\} \\
& <\exp \left\{h_{m}\left[\frac{1}{h_{m-1}}+\frac{\log h_{m}}{h_{m}}-\frac{1}{h_{n}}\left(1-\frac{1}{h_{n}}\right)\right]\right\} \\
& <\exp \left(-h_{m} / 2 h_{n}\right),
\end{aligned}
$$

and we see that $f^{\prime}(z) \sim a_{n+1} k_{n+1} z^{k_{n+1}-1}$ on the circle $|z|=r_{n}+1 / h_{n}{ }^{2}$. Because the estimate is also valid on the circle $|z|=r_{n+1}$, it holds throughout the domain between the $n$th and the $(n+1)$ st dirty annuli. Using the notation $f \#$ for the spherical derivative of $f$, we deduce that in the domain between the dirty annuli,

$$
\begin{aligned}
f \#(z)=\frac{\left|f^{\prime}(z)\right|}{1+|f(z)|^{2}} & <\left|f^{\prime}(z)\right| /|f(z)|^{2} \sim k_{n+1} /\left(a_{n+1}|z|^{k_{n+1}}\right) \\
& <h_{n+1}\left(\exp \frac{-h_{n+1}}{h_{n}}\right)\left(1-\frac{h_{n}-1}{h_{n}^{2}}\right)^{-h_{n+1}} \\
& =\exp \left\{\log h_{n+1}-\frac{h_{n+1}}{h_{n}}+h_{n+1}\left[\frac{h_{n}-1}{h_{n}^{2}}+\frac{1}{2}\left(\frac{h_{n}-1}{h_{n}^{2}}\right)^{2}+\ldots\right]\right\} \\
& \sim \exp \left\{\log h_{n+1}-\frac{h_{n+1}}{2 h_{n}^{2}}\right\}=o(1) .
\end{aligned}
$$

In other words, $f \sharp(z) \rightarrow 0$ as $z$ approaches the unit circle without entering any of the dirty annuli. This implies that if $r$ lies near 1 , then $f_{b}$ maps $\gamma_{r}$ onto a curve of small spherical length, except possibly for the portion of $\gamma_{r}$ whose image under $g_{b}$ falls into a dirty annulus.

Because the circle $g_{b}\left(\gamma_{r}\right)$ lies in the annulus with centre 0 and inner and outer radii

$$
\frac{|r-\beta|}{1-r \beta} \text { and } \frac{r+\beta}{1-r \beta}
$$

(notation of Section 8), and because $h_{n+1} / h_{n} \rightarrow \infty$ as $n \rightarrow \infty$, the circle $g_{b}\left(\gamma_{r}\right)$ meets at most one dirty annulus, if $r$ is sufficiently near 1 . Suppose that some points of $g_{b}\left(\gamma_{r}\right)$ lie in the $n$th dirty annulus. Then we can identify two maximal arcs $\gamma_{r}{ }^{*}$ and 
$\gamma_{r}{ }^{* *}$ such that $\left|g_{b}\left(r e^{i \theta}\right)\right|$ is monotone on each of the arcs and the image of each of the arcs lies in the dirty annulus. To be definite, we denote by $\gamma_{r}{ }^{*}$ the arc on which $\left|g_{b}(z)\right|$ is an increasing function of $\arg z$.

To facilitate the discussion of the spherical image of the $\operatorname{arc} \gamma_{r}{ }^{*}$, we denote by $\psi$ the stereographic projection of the plane onto the Riemann sphere. If the arc $g_{b}\left(\gamma_{r}{ }^{*}\right)$ begins at the inner boundary of the $n$th dirty annulus, then the behaviour of the function $\psi \circ f \circ g_{b}$ on the initial part of $\gamma_{r} *$ is determined almost entirely by the $n$th term in (1), and therefore the spherical image of the initial part of $\gamma_{r}{ }^{*}$ consists of smooth loops around the north pole, of approximate diameter $2 e / a_{n}$. As $g_{b}\left(\gamma_{r}{ }^{*}\right)$ penetrates deeper into the dirty annulus, the $(n+1)$ st term in (1) affects the curve $\psi \circ f \circ g_{b}\left(\gamma_{r}{ }^{*}\right)$ by generating shallow waves of high frequency. The amplitude of the waves increases gradually, and about at the image of the point on $\gamma_{r}{ }^{*}$ where

$$
\left|\left(a_{n+1} g_{b}^{k_{n+1}}\right)^{\prime}\right|=\left|\left(a_{n} g_{b}{ }^{k_{n}}\right)^{\prime}\right|,
$$

the waves change to loops. The loops increase in diameter until

$$
a_{n+1}\left|g_{b}{ }^{k_{n+1}}\right|=a_{n}\left|g_{b}{ }^{k_{n}}\right|
$$

then they begin to pass around the north pole, and as the term $a_{n} g_{b}{ }^{k_{n}}$ fades into relative insignificance, the loops become progressively smaller. The proof that the length of the $\operatorname{arc} \psi \circ f \circ g_{b}\left(\gamma_{r}{ }^{*}\right)$ is a bounded function of $r$ requires computations.

\section{A decomposition of $f_{b}$}

We write

$$
f_{b}=\sum_{m=1}^{n} a_{m} g_{b}^{k_{m}}+a_{n+1} g_{b}^{k_{n+1}}+\sum_{m=n+2}^{\infty} a_{m} g_{b}^{k_{m}}=f_{b 1}+f_{b 2}+f_{b 3} .
$$

Because both $f_{b 3}$ and $f_{b 3}^{\prime}$ are small in the $n$th dirty annulus, it follows from the triangle inequality that the effect of $f_{b 3}$ on the length of $\psi \circ f_{b}\left(\gamma_{r}{ }^{*}\right)$ is negligible; therefore we shall restrict our attention to the length of the arc $\psi \circ\left(f_{b 1}+f_{b 2}\right)\left(\gamma_{r}^{*}\right)$.

Although the first $n-1$ terms in the sum $f_{b 1}$ are much smaller than the $n$th term, we cannot ignore them; in regions where the sum $a_{n} g_{b}^{k_{n}}+a_{n+1} g_{b}^{k_{n+1}}$ is small, the first $n-1$ terms affect substantially the position of $f_{b}\left(\gamma_{r}{ }^{*}\right)$, and therefore they may affect the length of $\psi \circ f_{b}\left(\gamma_{r}{ }^{*}\right)$. But we note that

and

$$
f^{\prime}{ }_{b 2}=g_{b}^{\prime} g_{b}^{-1} k_{n+1} f_{b 2}
$$

$$
{f^{\prime}}^{\prime}{ }_{b 1}=g_{b}^{\prime} g_{b}^{-1} \sum_{1}^{n} a_{m} k_{m} g_{b}^{k_{m}} \sim g^{\prime}{ }_{b} g_{b}^{-1} k_{n} f_{b 1}
$$

so that

$$
\frac{f^{\prime}{ }_{b 2}}{f^{\prime}{ }_{b 1}} \sim \frac{h_{n+1}}{h_{n}} \frac{f_{b 2}}{f_{b 1}}
$$

This implies that at each point on $\gamma_{r}{ }^{*}$ one of the two quantities $\left|f_{b 2} / f_{b 1}\right|$ and $\left|f^{\prime}{ }_{b 2} / f^{\prime}{ }_{b 1}\right|$ lies outside the interval $[1 / 2,2]$.

It will be convenient to divide the $\operatorname{arc}{\gamma_{r}}_{r}^{*}$ into two $\operatorname{arcs} \lambda$ and $\mu$ (one of which may be empty). Recall that $\left|g_{b}\left(r e^{i \theta}\right)\right|$ is an increasing function of $\theta$ on $\gamma_{r}{ }^{*}$. 
If $\left|f_{b 2}(z)\right| \geqslant\left|f_{b 1}(z)\right|$ everywhere on $\gamma_{r}^{*}$, let $z_{0}=r e^{i \theta_{0}}$ denote the initial point of $\gamma_{r}{ }^{*}$, and if $\left|f_{b 2}(z)\right| \leqslant\left|f_{b 1}(z)\right|$ everywhere on $\gamma_{r}{ }^{*}$, let $z_{0}$ denote the terminal point of $\gamma_{r}{ }^{*}$. If neither of the inequalities holds everywhere on $\gamma_{r}^{*}$, let $z_{0}$ denote the first point on $\gamma_{r}{ }^{*}$ where $\left|f_{b 2}(z)\right|=\left|f_{b 1}(z)\right|$. Let $\lambda$ and $\mu$ denote the portions from the beginning of $\gamma_{r}{ }^{*}$ to $z_{0}$ and from $z_{0}$ to the end.

\section{The computations for $\mu$.}

As we pointed our near the the end of Section 9 , the image $\psi \circ f_{b}(\mu)$ consists of a sequence of progressively smaller loops around the north pole of the Riemann sphere. To estimate the combined length of these loops, we estimate first the position of the points $z_{j}=r e^{i \theta_{j}}(j=1,2, \ldots)$ on $\mu$ where

$$
\arg {f^{\prime}}^{\prime}(z)=\arg f^{\prime}{ }_{b 2}\left(z_{0}\right)+\pi j / 3
$$

for the first time. To a change $\Delta \theta$ in $\theta$ corresponds a change $\left(k_{n+1}+O(1)\right) \Delta \theta$ in $\arg f^{\prime}{ }_{b 2}(z)$, so that an increase $\pi / 3$ in $\arg f^{\prime}{ }_{b 2}(z)$ requires an increase greater than $A_{1} / k_{n+1}$ in $\theta$, where $A_{1}$ denotes a positive constant depending only on $|b|$.

On $\gamma_{r}{ }^{*}$ (and therefore on $\mu$ ) the modulus of $g_{b}\left(r e^{i \theta}\right)$ is an increasing function of $\theta$. The derivative of the modulus is 0 at $\theta_{0}$, if $g_{b}\left(z_{0}\right)$ is the point of $g_{b}\left(\gamma_{r}\right)$ nearest the origin; but even in that worst of all possible cases,

$$
\left|g_{b}\left(r e^{i \theta}\right)\right|>\left|g_{b}\left(r e^{i \theta_{0}}\right)\right|\left[1+A_{2}\left(\theta-\theta_{0}\right)^{2}\right]
$$

where $A_{2}$ is a positive constant proportional to the variation of $\left|g_{b}(z)\right|$ on the circle $\gamma_{r}$. Because this variation has the order of magnitude $1 / k_{n}$, we can assert that

$$
\left|g_{b}\left(z_{j}\right)\right|>\left|g_{b}\left(z_{0}\right)\right|\left(1+A_{3} j^{2} /\left(h_{n} h_{n+1}^{2}\right)\right),
$$

where $A_{3}$ again depends only on $|b|$.

It follows that

It follows that

$$
\begin{aligned}
\left|g_{b}\left(z_{j}\right)\right|^{k_{n+1}} & >\left|g_{b}\left(z_{0}\right)\right|^{k_{n+1}}\left(1+A_{3} j^{2} /\left(h_{n+1}^{2} h_{n}\right)\right)^{k_{n+1}} \\
& >\left|g_{b}\left(z_{0}\right)\right|^{k_{n+1}}\left(1+A_{3} j^{2} /\left(2 h_{n+1} h_{n}\right)\right) .
\end{aligned}
$$

$$
\left|g_{b}\left(z_{j}\right)\right|^{k_{n+1}}-\left|g_{b}\left(z_{0}\right)\right|^{k_{n+1}}>A_{3} j^{2}\left|g_{b}\left(z_{0}\right)\right|^{k_{n+1} /\left(2 h_{n+1} h_{n}\right)} .
$$

From this we deduce that

$$
\begin{aligned}
\left|f_{b 2}\left(z_{j}\right)\right|-\left|f_{b 2}\left(z_{0}\right)\right| & >A_{3} j^{2} a_{n+1}\left|g_{b}\left(z_{0}\right)\right|^{k_{n+1}} /\left(2 h_{n+1} h_{n}\right) \\
& =A_{3} j^{2}\left|f_{b 2}\left(z_{0}\right)\right| / 2 h_{n+1} h_{n} \\
& \geqslant A_{3} j^{2}\left|f_{b 1}\left(z_{0}\right)\right| /\left(2 h_{n+1} h_{n}\right) \\
& \sim A_{3} j^{2} a_{n} /\left(2 e h_{n+1} h_{n}\right) .
\end{aligned}
$$

The last expression provides the motivation for the relation (2) in Section 5. By virtue of that relation, we can now assert that

$$
\left|f_{b 2}\left(z_{j}\right)\right|-\left|f_{b 2}\left(z_{0}\right)\right|>A_{3} j^{2} / 6 .
$$

Because $\left|f_{b 2}\left(z_{j}\right)\right|>\left|f_{b 1}\left(z_{0}\right)\right|$ (see the end of Section 10), and because on the arc $\mu$ the response of $\left|f_{b 1}(z)\right|$ to an increase in $\left|g_{b}(z)\right|$ is much smaller than the response of 
$\left|f_{b 2}(z)\right|$, we can assert that on the $\operatorname{arc} \mu_{j}$ from $z_{j}$ to $z_{j+1}(j=1,2, \ldots)$,

$$
\left|f_{b}(z)\right|>A_{3} j^{2} / 7 \text {. }
$$

The referee believes that some readers will want to see analytical justification for the geometric argument in the preceding sentence. The authors thank the referee for supplying the following argument. We may assume that $z$ lies on the arc $\mu_{j}$ from $z_{j}$ to $z_{j+1}(j=1,2, \ldots)$. If we denote $g_{b}(z)-g_{b}\left(z_{0}\right)$ by $\delta$ and $\left|g_{b}(z)\right|-\left|g_{b}\left(z_{0}\right)\right|$ by $\eta$, then the argument at the beginning of this section shows that we have $\eta=O\left(j^{2} /\left(h_{n} h_{n+1}^{2}\right)\right)$. Moreover, it follows from (4) that

$$
\eta>A_{3} j^{2} /\left(2 h_{n} h_{n+1}^{2}\right), \text { while }|\delta|<B_{1} j / h_{n+1} .
$$

Thus $|\delta|<B_{2} \eta h_{n} h_{n+1}$. Let the function $\varepsilon($.) be defined by the formula

$$
f_{b 1}(z)=a_{n}\left\{g_{b}(z)\right\}^{k_{n}}\left[1+\varepsilon\left\{g_{b}(z)\right\}\right] .
$$

If $K$ is the maximum of $\left|\varepsilon^{\prime}(\zeta)\right|$ on the segment $\left[g_{b}\left(z_{0}\right), g_{b}(z)\right]$, we have the inequalities

$$
\begin{aligned}
\log \left|f_{b 1}(z)\right|-\log \left|f_{b 1}\left(z_{0}\right)\right| & <B_{3}\left\{h_{n} \eta+K|\delta|\right\} \\
& <B_{4} \eta\left\{h_{n}+K h_{n} h_{n+1}\right\} \\
& <B_{5} \eta\left\{h_{n}+\frac{h_{n} a_{n-1} h_{n} h_{n+1}}{a_{n}}\right\} \\
& =B_{5} \eta\left\{h_{n}+h_{n}{ }^{2} h_{n-1}\right\}<2 B_{5} \eta h_{n}{ }^{2} h_{n-1} .
\end{aligned}
$$

Using Lemma 1, we deduce that

$$
\log \left|f_{b 1}(z)\right|-\log \left|f_{b 1}\left(z_{0}\right)\right|=o\left(\eta h_{n+1}\right)=o\left(j^{2} /\left(h_{n} h_{n+1}\right)\right) .
$$

On the other hand, we deduce from (4) that

$$
\log \left|f_{b 2}(z)\right|-\log \left|f_{b 2}\left(z_{0}\right)\right|>\log \left(1+A_{3} j^{2} /\left(2 h_{n+1} h_{n}\right)\right) .
$$

We recall that $\left|f_{b 1}\left(z_{0}\right)\right|=\left|f_{b 2}\left(z_{0}\right)\right| \sim a_{n} / e$. Thus, when $n$ is large,

$$
\begin{aligned}
\log \left|f_{b 2}(z)\right|-\log \left|f_{b 1}(z)\right| & >\log \left(1+A_{3} j^{2} /\left(2 h_{n+1} h_{n}\right)\right)-\log \left(o\left(j^{2} /\left(h_{n+1} h_{n}\right)\right)\right) \\
& >\log \left(1+A_{3} j^{2} /\left(2.5 h_{n+1} h_{n}\right)\right) .
\end{aligned}
$$

Therefore,

$$
\begin{gathered}
\left|f_{b}(z)\right| \geqslant\left|f_{b 2}(z)\right|-\left|f_{b 1}(z)\right|-o(1) \geqslant\left|f_{b 1}(z)\right| A_{3} j^{2} /\left(2.5 h_{n+1} h_{n}\right)-o(1) \\
\sim a_{n} A_{3} j^{2} /\left(2.5 e h_{n+1} h_{n}\right)-o(1)>A_{3} j^{2} / 7 .
\end{gathered}
$$

LEMMA 2. If at each point of some differentiable arc $C$ in the w-plane, the tangent line to $C$ makes an angle at most $\pi / 4$ with some fixed line $S$, and if the distance between $C$ and the origin is $\delta$, then the spherical length of $C$ is less than $6 \operatorname{arccot} \delta$.

Proof. After a rotation, we may assume that $S$ is the real line. Let $C_{1}, C_{2}, C_{3}$, and $C_{4}$ denote the portions of $C$ lying in the half-plane $u \leqslant-\delta / \sqrt{ } 2$, in the strip $-\delta / \sqrt{ } 2 \leqslant u \leqslant 0$, in the strip $0 \leqslant u \leqslant \delta / \sqrt{ } 2$, and in the half-plane $u \geqslant \delta / \sqrt{ } 2$, respectively.

If $C_{4}$ is not empty, let $t$ denote arc length on $C_{4}$, measured from left to right. The inequality $d u / d t \geqslant 1 / \sqrt{ } 2,(w=u+i v)$, implies that the spherical length of $C_{4}$ is 
at most

$$
\begin{gathered}
\int_{0}^{\infty} \frac{d t}{1+u^{2}} \leqslant \int_{0}^{\infty} \frac{d t}{1+(\delta+t)^{2} / 2} \\
=\left.\sqrt{ } 2 \arctan \frac{\delta+t}{\sqrt{ } 2}\right|_{0} ^{\infty}=\sqrt{ } 2 \arctan \frac{\sqrt{ } 2}{\delta} .
\end{gathered}
$$

Because on the positive half-line the arctangent is a concave function,

$\arctan \alpha x<\alpha \arctan x$

where $\alpha>1$, and therefore the spherical length of $C_{4}$ is less than

$$
2 \arctan 1 / \delta=2 \operatorname{arccot} \delta .
$$

If $C_{3}$ is not empty, let $s$ denote arc length on $C_{3}$. Because $0 \leqslant s \leqslant \delta$ on $C_{3}$, the spherical length of $C_{3}$ is not greater than

$$
\int_{0}^{\delta} \frac{d s}{1+\delta^{2}}=\frac{\delta}{1+\delta^{2}}=\frac{1}{\delta} \frac{1}{1+1 / \delta^{2}} \leqslant \int_{0}^{1 / \delta} \frac{d x}{1+x^{2}}=\operatorname{arccot} \delta .
$$

Analogous computations yield similar bounds for the spherical length of $C_{1}$ and $C_{2}$, and Lemma 2 follows immediately.

Returning to the task of estimating the spherical length of $f_{b}(\mu)$, we recall that on each of the $\operatorname{arcs} \mu_{j}(j=0,1, \ldots)$ the variation of $\arg {f^{\prime}}_{b 2}(z)$ is $\pi / 3$. Because ${f^{\prime}}_{b 1}(z)$ and $f^{\prime}{ }_{b 3}(z)$ are much smaller than ${f^{\prime}}_{b 2}(z)$, the values of $\arg {f^{\prime}}_{b}(z)$ lie in an interval whose length is only slightly greater than $\pi / 3$. Moreover, on each arc $\mu_{j}$ the argument of $d w / d z$ is restricted to a short interval, and therefore we can apply Lemma 2 and deduce that for all values $r$ sufficiently near 1 , the spherical length of $f_{b}(\mu)$ is less than

$$
6 \sum_{0}^{\infty} \operatorname{arccot} A_{3} j^{2} / 7
$$

Because of the constant $A_{3}$, the value of this bound depends on $|b|$; but it is independent of $r$, and therefore the spherical length of $f_{b}(\mu)$ is a bounded function of $r$.

\section{The computations for the arc $\lambda$.}

The $\operatorname{arc} \psi \circ f_{b}(\mu)$ studied in Section 11 winds around the Riemann sphere's north pole in progressively tighter loops. The arc $\psi \circ f_{b}(\lambda)$, which will occupy us now, consists largely of loops about a centre that moves slowly at a distance approximately $e / a_{n}$ from the north pole.

First we dispatch the portion of $\lambda$ where

$$
a_{n+1} h_{n+1}\left|g_{b}\right|^{h_{n+1}} \leqslant 2 a_{n} h_{n}\left|g_{b}\right|^{h_{n}} .
$$

Here $\left|f_{b 2}^{\prime}\right|<3\left|f_{b 1}^{\prime}\right|$ and $f_{b 2} / f_{b 1}$ is small. Consequently,

$$
f_{b} \#<5\left|f^{\prime}{ }_{b 1} / f_{b 1}{ }^{2}\right| \sim 5 h_{n} /\left|f_{b 1}\right| \sim 5 e h_{n} / a_{n}=5 e / h_{n+1} .
$$

Therefore $f_{b}$ maps this portion of $\lambda$ onto an arc of small spherical length. 
Next we deal with the portion where

$$
2 a_{n} h_{n}\left|g_{b}\right|^{h_{n}} \leqslant a_{n+1} h_{n+1}\left|g_{b}\right|^{h_{n+1}} \leqslant a_{n} h_{n+1}\left|g_{b}\right|^{h_{n}} / 2 \text {. }
$$

The second inequality implies that $\left|f_{b}\right|>\left|f_{b 1}\right| / 3$, and therefore, by virtue of the first inequality,

$$
f \sharp_{b}<\frac{2\left|f_{b 2}^{\prime}\right|}{\left|f_{b 1} / 3\right|^{2}} \sim \frac{18 h_{n+1}\left|f_{b 2}\right|}{\left|f_{b 1}\right|^{2}}<18 h_{n+1} /\left|f_{b 1}\right| \sim 18 e h_{n+1} / a_{n}=18 e / h_{n} .
$$

Again, we conclude that $f_{b}$ maps the portion of $\lambda$ onto an arc of small spherical length.

This leaves us with the portion of $\lambda$ where

$$
a_{n+1}\left|g_{b}\right|^{h_{n+1}} \geqslant a_{n}\left|g_{b}\right|^{h_{n}} / 2 \text {. }
$$

We recall that, by virtue of the definition of $\lambda$,

$$
a_{n+1}\left|g_{b}\right|^{h_{n+1}} \leqslant\left|f_{b 1}\right|
$$

so that now $\left|f_{b 2}\right|$ lies between $\left|f_{b 1}\right|$ and approximately $\left|f_{b 1}\right| / 2$. We use the remainder of this section to show that $f_{b}$ maps the portion of $\lambda$ determined by (5) onto a curve whose spherical length has an upper bound independent of $r$.

We assign to $\arg \left\{f_{b 2}\left(z_{0}\right) / f_{b 1}\left(z_{0}\right)\right\}$ its lowest possible positive value; for $j=0,1, \ldots$ we define the points $t_{j}=r e^{i \tau_{j}}$ on $\lambda$ by the condition

$$
\arg \left\{f_{b 2}\left(t_{j}\right) / f_{b 1}\left(t_{j}\right)\right\}=-(2 j+1) \pi,
$$

and we denote by $\lambda_{j}(j=0,1, \ldots)$ the portion of $\lambda$ between $t_{j}$ and $t_{j+1}$ (except that the last of the arcs $\lambda_{j}$ is the arc from the corresponding point $t_{j}$ to the point where

$$
\left.\left|f_{b 2}\right|=a_{n}\left|g_{b}\right|^{h_{n}} / 2\right) \text {. }
$$

Because $\arg {f^{\prime}}_{b 2}$ is monotone on $\lambda$ and has values restricted to the interval $[-3 \pi, 2 \pi]$ on the arc from $z_{0}$ to $t_{1}$, and because the variation of $\arg f^{\prime}{ }_{b 1}$ on that arc is small, we can divide the arc from $z_{0}$ to $t_{1}$ into eleven subarcs on each of which $\arg f^{\prime}{ }_{b}$ is restricted to an interval of length less than $\pi / 2$. To each of the eleven subarcs we apply Lemma 2 of Section 11.

On each of the $\operatorname{arcs} \lambda_{j}(j=1,2, \ldots)$, the ratio $f^{\prime}{ }_{b} / f^{\prime}{ }_{b 2}$ is nearly 1 , and therefore the direction of the tangent line to the curve $f_{b}\left(\lambda_{j}\right)$ differs only slightly from the direction of the tangent line at the corresponding point of the curve defined by the equation

$$
w(z)=a_{n+1}\left(g_{b}(z)\right)^{k_{n+1}} .
$$

In other words, the curve $f_{b}(\lambda)$ consists of smooth loops. Our next task is to prove that the distance between the loop $f_{b}\left(\lambda_{j}\right)$ and the origin is fairly large. then

Reasoning as in the first two paragraphs of Section 11, we see that if $\tau_{j} \geqslant \theta \geqslant \tau_{j+1}$,

$$
\left|g_{b}\left(r e^{i \theta}\right)\right|<\left|g_{b}\left(t_{0}\right)\right|\left(1-A_{4} j^{2} / h_{n} h_{n+1}^{2}\right)
$$

in other words, that

$$
\left|g_{b}\left(r e^{i \theta}\right)\right|^{k_{n+1}} \leqslant\left|g_{b}\left(t_{0}\right)\right|^{k_{n+1}}\left(1-A_{4} j^{2} / a_{n} h_{n+1}\right)^{k_{n+1}} .
$$

As we remarked after (5), $\left|f_{b 2}\right|$ lies between $\left|f_{b 1}\right|$ and approximately $\left|f_{b 1}\right| / 2$, so that the second factor on the right is larger than $2 / 5$, say. Now if $0<u<1$ and $x>1$, 
and if

$$
F(u)=(1-u)^{x}>2 / 5,
$$

it follows from the mean-value theorem that

$$
F(u)-F(0)=-u x\left(1-u_{0}\right)^{x-1}
$$

for some $u_{0}$ in the interval $(0, u)$. It follows that

$$
1-(1-u)^{x}=u x\left(1-u_{0}\right)^{x-1}>u x(1-u)^{x-1}>u x(1-u)^{x}>2 u x / 5,
$$

and therefore

$$
(1-u)^{x}<1-2 u x / 5
$$

Setting $u=A_{4} j^{2} / a_{n} h_{n+1}$ and $x=k_{n+1}$, we deduce that

$$
\left(1-A_{4} j^{2} / a_{n} h_{n+1}\right)^{k_{n+1}}<1-2 A_{4} j^{2} k_{n+1} / 5 a_{n} k_{n+1}<1-\frac{A_{4} j^{2}}{3 a_{n}}
$$

and hence that

$$
\left|g_{b}\left(t_{0}\right)\right|^{k_{n+1}}-\left|g_{b}\left(t_{j}\right)\right|^{k_{n+1}} \geqslant\left|g_{b}\left(t_{0}\right)\right|^{k_{n+1}} A_{4} j^{2} /\left(3 a_{n}\right) .
$$

Because $f_{b 2}=a_{n+1} g_{b}^{k_{n+1}}$, we can write this in the form

$$
\left|f_{b 2}\left(t_{0}\right)\right|-\left|f_{b 2}\left(t_{j}\right)\right|>\left|f_{b 2}\left(t_{0}\right)\right| A_{4} j^{2} /\left(3 a_{n}\right),
$$

and by (5), the right hand member is greater than

$$
a_{n}\left|g_{b}\left(t_{0}\right)\right|^{h_{n}} A_{4} j^{2} /\left(6 a_{n}\right) \sim A_{4} j^{2} /(6 e) .
$$

Because on $\lambda_{j}$ the derivative of $\left|f_{b 2}\right|$ with respect to arc length is much larger than the derivative of $\left|f_{b 1}\right|$, and because the spherical variation of $f_{b 1}$ on $\lambda_{j}$ is much smaller than $1 / a_{n}$, the length of $\psi \circ f_{b}\left(\lambda_{j}\right)$ is not greater than twice the length of the spherical image of the circle with centre $f_{b 1}\left(t_{j}\right)$ and radius $\left|f_{b 2}\left(t_{j}\right)\right|$. A diameter of that circle is a radial segment whose endpoints have modulus

$$
\left|f_{b 1}\left(t_{j}\right)\right|-\left|f_{b 2}\left(t_{j}\right)\right| \text { and }\left|f_{b 1}\left(t_{j}\right)\right|+\left|f_{b 2}\left(t_{j}\right)\right| .
$$

The chordal distance between two points $w_{1}$ and $w_{2}$ is

$$
\left|w_{1}-w_{2}\right| / \sqrt{ }\left(1+\left|w_{1}\right|^{2}\right) \quad \sqrt{ }\left(1+\left|w_{2}\right|^{2}\right)
$$

suppressing the variable $t_{j}$, we can therefore write the spherical length of our circle in the form

$$
2 \pi\left|f_{b 2}\right| / \sqrt{ }\left(1+\left(\left|f_{b 1}\right|-\left|f_{b 2}\right|\right)^{2}\right) \quad \sqrt{ }\left(1+\left(\left|f_{b 1}\right|+\left|f_{b 2}\right|\right)^{2}\right) .
$$

Obviously, this is less than

$$
2 \pi /\left(\left|f_{b 1}\left(t_{j}\right)\right|-\left|f_{b 2}\left(t_{j}\right)\right|\right) \text {. }
$$

Because $\left|f_{b 1}\left(t_{j}\right)\right|-\left|f_{b 2}\left(t_{j}\right)\right| \sim\left|f_{b 1}\left(t_{0}\right)\right|-\left|f_{b 2}\left(t_{j}\right)\right|$ and $\left|f_{b 1}\left(t_{0}\right)\right| \geqslant\left|f_{b 2}\left(t_{0}\right)\right|$, it follows from an argument like that in the preceding section that the spherical length of $f_{b}\left(\lambda_{j}\right)$ is less than $A_{s} / j^{2}$. This concludes the proof that $f_{b}$ is a Tsuji function.

\section{The Julia points of $f_{b}$}

On both boundary components of the $n$th dirty annulus, the modulus of $f(z)$ is at least $a_{n} / 3$. The same is true on the radii (in the annulus) on which $\left(k_{n+1}-k_{n}\right)$ $\arg z$ is an even multiple of $\pi$. On the radii on which $\left(k_{n+1}-k_{n}\right) \arg z$ is an odd 
multiple of $\pi$, there exists a point in the $n$th dirty annulus $(n=1,2, \ldots)$ where the sum of the $n$th and $(n+1)$ st terms of (1) is 0 , and at this point $|f(z)|<2 a_{n-1}$. By Rouchés theorem, $f(z)$ assumes equally often (and therefore at least once) all values of modulus less than $a_{n} / 3$, in each sector of the $n$th dirty annulus determined by a relation of the form

$$
2 \pi \frac{m-1}{k_{n+1}-k_{n}}<\arg z<2 \pi \frac{m}{k_{n+1}-k_{n}}(m=0,1, \ldots) .
$$

Clearly, the spherical image of each sector covers the Riemann sphere, except for a small neighbourhood of the north pole. Because the diameter of the sector is small compared with its distance from the unit circle, it follows that each point $e^{i \theta}$ is a Julia point of $f$.

If each point $e^{i 0}$ is a Julia point of $f$, then clearly each point $e^{i \theta}$ is a Julia point of $f_{b}$. This concludes the proof of Theorem 1 .

\section{The Julia points of functions in Hayman's class $T_{2}$}

We conclude with a proof that for every annular Tsuji function, the set of Julia points is large in both the topological and the measure-theoretic sense.

E. F. Collingwood [3] proved that if $f$ is a meromorphic Tsuji function in D, then almost every point on the unit circle $C$ is either a Fatou point or a Julia point for $f$. Because the Fatou points of an annular function constitute a set of measure 0 , the set of Julia points of an annular Tsuji function must have measure $2 \pi$. That it is also a residual subset of $C$ follows immediately from our second theorem. The theorem deals with a class of functions more general than the class of Tsuji functions.

Following Hayman, we say that a meromorphic function $f$ in $D$ belongs to the class $T_{2}$ provided there exists a sequence $\left\{J_{n}\right\}$ of Jordan curves whose interiors expand to $D$ (in the sense of Section 1) and whose images $f\left(J_{n}\right)$ have bounded spherical length. We denote by $J(f)$ the set of Julia points of $f$, and we say that a point of $C$ belongs to the set $X(f)$ provided it is the midpoint of some arc on $C$ to which $f$ has a continuous extension (in the spherical metric). With this notation, our result takes the following form.

THEOREM 2. If $f \in T_{2}$, then $X(f) \cup J(f)$ is residual on $C$.

Proof. Let $N(f)$ denote the set of points on $C$ where $f$ is normal, that is, the set of points $e^{i \theta}$ to which there corresponds a pair of positive numbers $r$ and $M$ such that

$$
f \#(z)<M /(1-|z|)
$$

whenever $z \in D$ and $\left|z-e^{i \theta}\right|<r$. Because $f$ is meromorphic, it follows from results of S. Dragosh [5; Theorems 8 and 9] that the set $N(f) \cup J(f)$ is residual on $C$. Furthermore, Hayman [7; Theorem 3] has proved that $N(f)=X(f)$, for functions $f$ in $T_{2}$; this proves Theorem 2 .

Theorem 2 contradicts the statement at the end of page 199 of Collingwood's paper [3]. Collingwood stated that even if a Tsuji function has no Fatou points, the set of its Julia points may still be of first category. The proposed proof is based on an 
example furnished by Piranian in an informal communication. Piranian regrets that his construction involves an error: the first clause in the final sentence (middle of page 200) in [3] is incorrect.

\section{References}

1. D. D. Bonar, F. W. Carroll and P. Colwell, "Category results for Tsuji functions ", Canad. J. Math., 29 (1977), 552-558.

2. D. D. Bonar, F. W. Carroll and G. Piranian, "Strongly annular functions with small Taylor coefficients ", Math. Z., 156 (1977), 85-91.

3. E. F. Collingwood, "A boundary theorem for Tsuji functions ”, Nagoya Math. J., 29 (1967), 197-200.

4. E. F. Collingwood and G. Piranian, “"Tsuji functions with segments of Julia ”, Math.Z., 84 (1964), 246-253.

5. S. Dragosh, "The spherical derivative of meromorphic functions", J. Reine Angew. Math., 252 (1972), 51-67.

6. W. K. Hayman, "Regular Tsuji functions with infinitely many Julia points ", Nagoya Math. J., 29 (1967), 185-196.

7. W. K. Hayman, “The boundary behaviour of Tsuji functions”, Michigan Math. J., 15 (1968), $1-26$.

8. R. W. Howell, "Annular functions in probability ", Proc. Amer. Math. Soc., 52 (1975), 217-221.

Pennsylvania State University, University Park, Pennsylvania 16802.

Denison University, Granville, Ohio 43023.

Ohio State University, Columbus, Ohio 43210.

Iowa State University, Ames, Iowa 50011.

University of Michigan, Ann Arbor, Michigan 48109. 